changes to be confined to the anterior segment of the eye. Possibly special methods of staining might show degeneration of the ganglion cells.

The two cases are apparently the two extremes of what we know as sympathetic disease. In one the inflammation is of the septic type; characterised by leucocytic extravasation, and some lymphocyte collections, in the other by the absence of leucocytes, but with some lymphatic collections and with much plasma cell formation. The common type shows mainly lymphocyte nodules.

One would hope that the third case is unique. To find destructive sympathetic disease following a successful operation for cataract, where the only evidence of inflammation was the presence of a few precipitates on Descemet's membrane which were visible for a few weeks at most is fortunately unusual.

It is, however, evident from the case E. B., where a possibly dangerous eye was removed, that one does not do all that is necessary unless one makes a careful slit-lamp examination of the remaining eye before the case leaves hospital, and again afterwards till safety is assured. The same would hold good for any postoperative evidence, however slight, if iridocyclitis is present, though in a case like my last it is difficult to see what one could have done to prevent trouble, in the face of the fact that the exciting eye was and is free from disease.

\title{
AN UNUSUAL CASE OF NEURO-EPITHELIOMA OF THE RETINA
}

BY

\author{
D. G. Patwardhan, D.O.(Oxon.), D.O.M.S.(Lond.), \\ M.B., B.S.(Bomb.) \\ OPHTHALMOLOGIST, B.J. MEDICAL SCHOOL \\ POONA
}

THE patient, a boy aged 6 years, was brought to Poona, Sassoon Hospital, and was admitted for treatment on June 25, 1929.

State on admission. - The patient had a tumour of the left eyeball of the size of a coconut and he complained of extreme pain in that region. He was not emaciated and the state of his health appeared to be fairly normal.

History of the case.-About six months previously the patient had an injury to the left eyeball. The eyeball was perforated and there was a good deal of haemorrhage. There was no help available from a doctor and hence he was treated by homely 
remedies. The bleeding stopped and the wound healed for all practical purposes. The patient lost his eyesight. After about a week or so there appeared a swelling at the site of the injury of the size of a ground-nut. This swelling went on increasing gradually until it attained its present size. All sorts of medicines were tried

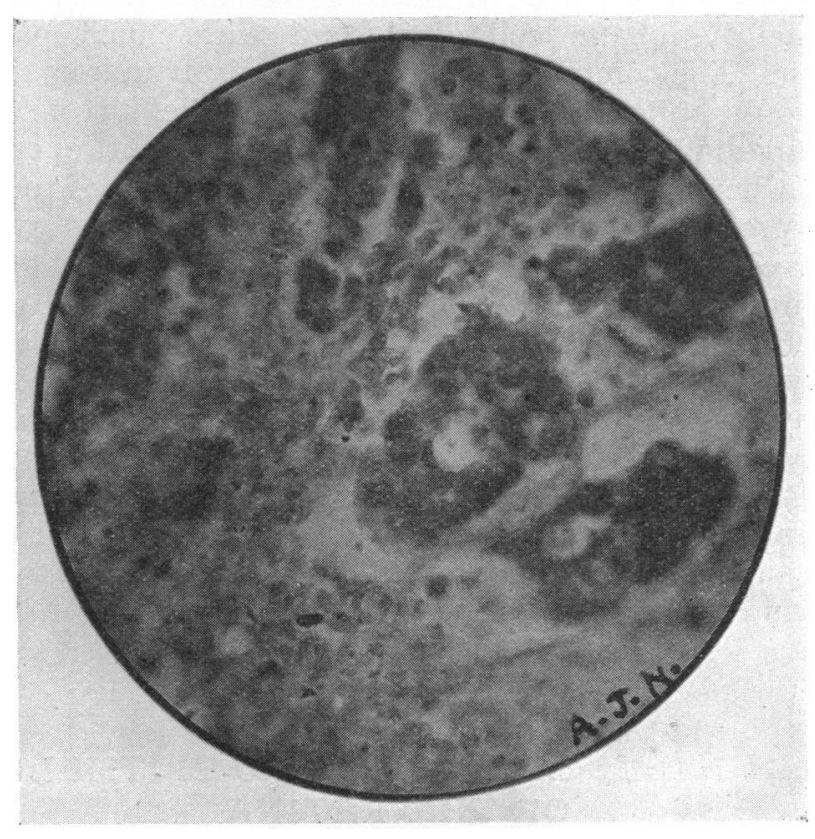

Photograph showing masses of cells surrounding lumina that do not contain any blood. They seem to represent badly formed rosettes found in tumours of slower growth.

without avail. The pain was increasing along with the increase in the size of the tumour.

On examination it was found that the swelling was as big as a coconut covering the area from the nose on the inner side to the tragus of the left ear on the outer side, and from about an inch above the orbital margin low down to the angle of the jaw. The actual measurements were from side to side 10 inches; from above downwards 9 inches. The circumference was 15 inches. The tumour had a stalk originating from in between the eyelids and it was not adherent to any of the surrounding structures. It had a very foul smell and its consistency was.as soft as brain. The appearance was lobulated with depressions in between which were areas studded with yellowish looking material. In some places 
bleeding points could be seen. There was no enlargement of the glands of the neck. The fundus of the other eye was perfectly normal and the media clear. The vision of the sound eye was $6 / 6$.

$X$-ray Report.-No secondary deposits in the viscera were found. The X-ray photograph of the skull did not reveal any shadow.

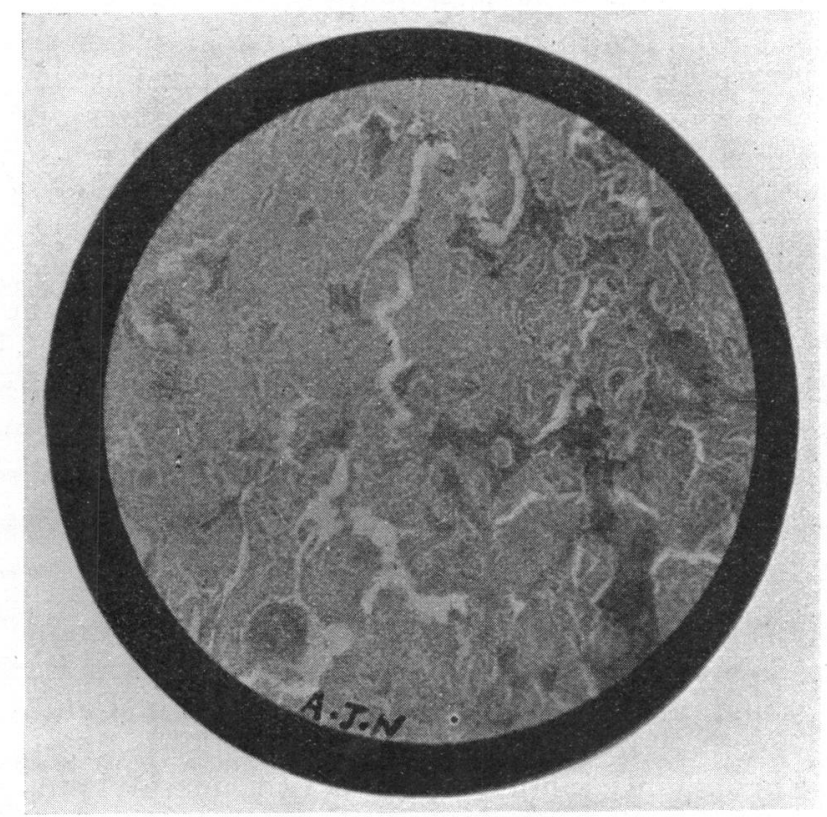

Low power photograph showing the growing edge of the tumour, profuse haemorrhages present, cellular masses traversed by sinuses.

A photograph was taken from the front as well as sideways. The tumour was removed under chloroform on July 2, 1929, and exenteration of the left orbit was done. There was not much bleeding during the operation. The weight of the tumour was taken after its removal and was found to be 620 grammes. The tumour as well as the exenterated material was sent for the pathologist's report.

Clinical diagnosis.-Glioma (exophytum) of the retina.

Pathological findings.-To the naked eye the tumour is a fungating cauliflower-like mass, soft and somewhat elastic to the touch, with elevations and depressions on the surface which here and there shows signs of ulceration. There are clearly discernible 
bleeding points on the surface and also sticky pus-like material, especially in the furrows. In size the tumour is larger than a fist and there is on the under surface the mark indicating the division of what looks like a pedicle.

Microscopically one can recognise areas of degeneration in the midst of fairly well preserved tissue which gets scantier towards the central portion of the growth, as judged from sections taken from this part. The undegenerated portions consist of cells poor in protoplasm and with deeply staining nuclei which are oval or rounded. Mitotic figures of various kinds have been observed. The cellular masses are traversed by blood sinuses and profuse haemorrhages are present. The degenerated portions show " ghosts" of cells with nuclei either entirely unstained or slightly so, being in places piknotic. Islets of fairly well preserved cells are discernible here and there in these areas, arranged around lumina that do not contain any blood. One fails to find regular " rosettes." There are here and there cells with large elongated ovoid faintly-staining nuclei with fibrils and here and there endothelial cells are present. In the neighbourhood of ulcerated areas polynuclear leucocytes are found penetrating the substance of the growth.

Diagnosis.-Neuro-epithelioma of the retina.

The only peculiarity in the case in point was the unusual size of the tumour and the short period during which it attained such a huge size. Similar cases are reported in "Surgical Pathology," by McFarland, and in Banerjee's " Text Book of Pathology."

I am indebted to Dr. Noronha, Pathologist, B. J. Medical School, for having kindly lent me two micro-photographs of sections of the tumour.

\section{ANNOTATION}

\section{The other half of the Story}

It was at one time the habit of a certain section of the legal community to concoct an apparently impossible story and demand some rational explanation of the sequence of events. The best known one, reputed to be true, concerns a girl and her mother who visited Paris at the time of the great exhibition. They had come from abroad and took rooms at an hotel where they had dinner together. Their rooms were next door to each other, and they retired to bed at about 10 o'clock in the evening. When the daughter awoke next morning she tried to go to her mother's room 